\title{
Prioritization of Alternative Agricultural Prod- ucts Based on Market Signals as a Tool for the Development of Innovative Projects
}

\section{Priorización de Productos Agrícolas Alterna- tivos en Base a Señales de Mercado Como Herramienta para Desarrollo de Proyectos Innovadores}

VII International Congress of

Science, Technology,

Entrepreneurship and

Innovation (SECTEI 2020)

Corresponding Author:

M. F. Logroño-Rodríguez

aledzoe@gmail.com

Published: 26 August 2021

Production and Hosting by

Knowledge $E$

(c) D. Villalva-Bustamante et al. This article is distributed under the terms of the Creative Commons Attribution License, which permits unrestricted use and redistribution provided that the original author and source are credited.

\author{
D. Villalva-Bustamante ${ }^{1}$, M. F. Logroño-Rodríguez ${ }^{2}$, T. F. Flores-Pulgar ${ }^{2}$, and B. \\ Naula-Erazo ${ }^{1}$ \\ ${ }^{1}$ Escuela Superior Politécnica de Chimborazo, Riobamba, Ecuador \\ ${ }^{2}$ Instituto Superior Pedagógico Intercultural Bilingüe Jaime Roldós Aguilera, Ecuador
}

\section{Abstract}

The Decentralized Autonomous Government of the province of Chimborazo promotes the agricultural productive development of the province by promoting entrepreneurship, and improving the capacities of producers, access to production factors, and integration of actors and subjects of agriculture and management on the basis to strategic planning. The construction of the Chimborazo Competitiveness Agenda was a participatory process that identified opportunities to improve the agricultural sector, where agricultural production at the primary level was recognized as a vocation and strength of the province; however, a strategic necessity was to establish possibilities of adding value and diversifying agricultural production. The definition of a set of agricultural products with high potential at the primary level and/or in agroindustrial processes that are not deeply explored required determining market signals to identify new medium- and long-term commercial possibilities for Chimborazo farmers. Additionally, it was recognized that the management of the province of Chimborazo was based on its management of the Millennium Development Goals, the Constitution of the Republic, the National Plan for Development in a Supra-Provincial Framework, as well as the Plan Development of Chimborazo, the Territorial Planning Plan and others that promoted planning with a focus on territorial and solidarity sustainable development.

Keywords: Chimborazo, productive development, entrepreneurship, agricultural sector

\section{Resumen}

El Gobierno Autónomo Descentralizado de la Provincia de Chimborazo impulsa el fomento productivo agrícola de la provincia mediante la promoción del emprendimiento, el mejoramiento de las capacidades de los productores, acceso a factores de producción, integración de los actores y sujetos del agro y gestión en base a una planificación estratégica. Como parte de dicho accionar, la construcción de la Agenda de Competitividad Chimborazo fue un proceso participativo que identificó oportunidades para mejorar el sector agrícola, donde se reconoció la producción agrícola a nivel primario como una vocación y fortaleza de la provincia, no obstante, una necesidad estratégica fue el establecer posibilidades de agregación de valor y de diversificar la producción agrícola. La definición de un conjunto de productos agrícolas con alto potencial a nivel primario y/o en procesos agroindustriales que 
no están profundamente explorados requirió determinar señales de mercado para identificar posibilidades comerciales nuevas a mediano y largo plazo para los agricultores de Chimborazo. Adicionalmente, se reconoció que la gestión de la provincia de Chimborazo tuvo como base los Objetivos del Milenio, la Constitución de la República, el Plan Nacional de Desarrollo en un marco supra-provincial, así como el Plan de Desarrollo de Chimborazo, el Plan de Ordenamiento Territorial y otros en su conjunto que promovieron una planificación con un enfoque de desarrollo sostenible territorial y solidario.

Palabras Clave: Chimborazo, fomento productivo, emprendimiento, sector agrícola.

\section{Introducción}

Previo al boom petrolero, el Ecuador se caracterizaba por la producción agrícola de exportación de cacao y banano, productos de clima cálido; para los años 90 llega el boom de las flores a la región sierra norte, situación que a la par condujo a establecer espacios de agro-exportación para productos no tradicionales. Esto deja ver que las ventajas de la región costa para su desarrollo de agroexportador fueron sus puertos, entre tanto los aeropuertos cumplieron su rol de conexión directa entre mercado de demanda y los productores de la región sierra. Sin embargo la provincia de Chimborazo, por la falta de puntos de embarque que conecten con mercados externos, únicamente profundizó su fuerza agrícola para satisfacer la necesidad de alimentos de los polos económicos nacionales que son Guayaquil y Quito principalmente.

Además es relevante recordar que la realidad agrícola de Chimborazo estuvo condicionada en los últimos 40 años por una concentración de la propiedad de la tierra productiva en pocas manos, situación que excluyo y abuso de una alta demografía campesina e indígena, marcando la pobreza en el área rural y debilitando la economía campesina de la agricultura familiar. Los efectos visibles son las transformaciones ambientales de los recursos, una alta densidad demográfica rural frente a la insuficiente tierra de producción, lo que han conllevado a un proceso permanente de abandono del campo, repercutiendo en la baja productividad y competitividad de una provincia de alta vocación y herencia agrícola [1].

Los trabajadores del campo que aún persisten trabajando la tierra dan muestras de un sentir de inviabilidad agrícola, esto cuando reconocen que no hay un pago justo por sus cosechas, cuando no encuentran acceso a los factores productivos agrícolas, cuando no se reconoce su trabajo y esfuerzo de sostener la alimentación (subsidiar), especialmente a las zonas urbanas. Es evidente la opción que han tomado muchos campesinos e indígenas, de cambiar los campos agrícolas por pastos y ganado lechero, el cual demanda menor mano de obra y los beneficios son a muy corto plazo, en 
otros casos, en un menor número tiendo la oportunidad de riego, financiamiento y conocimientos, han trascendido a explorar productos agrícolas y forestales alternativos, los cuales son demandados por mercados no convencionales. Siendo de estos últimos a los que se busca promover en su diversificación productiva.

De este modo los esfuerzos institucionales de los últimos años, al respecto del agro, vienen buscando menguar los efectos de una subestimación del sector agrícola, al tiempo de encontrar mecanismos de diversificación, agregación de valor, calidad, productividad y competitividad para las cadenas productivas. Dicho sentido implica establecer una línea de conexión entre los productores y los mercados, lo cual conlleva a identificar los cultivos de oportunidad que se desarrollan en la provincia no únicamente desde la producción primaria y/o de materias primas, sino de sus derivados; a identificar las desventajas de las condiciones de producción rustica, con poca tecnología y de baja innovación que no permite disminuir los costos frente a una alta competitividad; a identificar los sistemas de comercialización y las estrategias apropiadas de llegar a los consumidores.

En materia de comercio, no hay que perder de vista que la oferta de productos agrícolas juega un rol importante, pero que debe estar marcado por el valor agregado como la clave para llegar a satisfacer la demanda de los mercados nacionales e internacionales. No obstante los conceptos de consumo han evolucionado, relegando a la producción denominada tradicionales frente a una sustitutiva o 'Innovadora' que tenga atributos y características como saludable, dietético, light, entre otros en el caso de los alimentos. Estos enfoque provenientes de la globalización de los mercados, apuestan siempre a satisfacer amplios segmentos de demandas insatisfechas, y para lograrlo se requiere volúmenes de mayor cosecha, lo que conduce a provocar monocultivos especializados en función de contar con mano de obra calificada en conocimiento, manejar tecnología e insumos especializado, que a su vez resulta costoso, sumando las operaciones de transformación, trasporte de materias primas en condiciones de reducir los aspectos perecederos y ampliar el tiempo de uso comercial.

El Gobierno Autónomo Descentralizado (GAD) de la Provincia de Chimborazo reconoce la necesidad de generar políticas públicas y plantear planes de acción que motiven y fortalezcan las capacidades innovadoras de los agricultores, especialmente en función de los pequeños productores y desde su agrobiodiversidad, para ello se ha propuesto un estudio que identifique señales de mercado de un listado de 16 productos de características innovadores que tienen posibilidades de convertirse en adecuados complementos para la producción tradicional, los cuales fueron propuestas por los propios agricultores en la construcción de la Agenda de Competitividad. 


\section{Metodología}

La identificación de señales de mercado, planteadas por el GAD de Chimborazo, se entiende por el propósito de encontrar caminos que conduzcan una producción específica de la provincia a mercados estratégicos. En tal sentido, para un entendimiento más amplio se brinda soportes técnicos de enfoque y pensamiento respecto de la creciente integración de la economía que reduce las barreras a escala global, donde la producción de bienes y servicios de muchos micro empresarios, y también de pequeños productores, es atraída por la necesidad de encontrar las oportunidades de insertarse en mercados crecientes de demandas insatisfechas, claro está, con el fin de obtener mayores ingresos.

Este caminar a nuevos mercados y de posibilidades de satisfacer las necesidades del consumidor final, requiere de una sensibilidad por la productividad y también por la competitividad, lo cual se traduce en lógicas de mejoramiento de capacidades de inversión con innovación y calidad, alta capacidad del talento humano, el desarrollo de procesos estratégicos de comercialización, la generación de enlaces con mercados de consumo, sin perder de vista la relación: Producción-agregación de valorcomercialización-consumo.

Se establece un marco de concepciones que permitan no solo identificar a dónde llegar, sino principalmente a entender el camino por el cual se deberá transitar.

\subsection{La provincia de Chimborazo}

\subsubsection{Extensión y ubicación}

La Provincia de Chimborazo tiene una extensión de 6.495 Km2. Se ubica en la sierra central del Ecuador, limita al Norte con la provincia de Tungurahua, al Sur con la provincia de Cañar, al Este con la provincia de Morona Santiago y al Oeste con las provincias de Guayas y Bolívar. Las ciudades más cercanas a la provincia son Ambato a 47 km, Quito a $191 \mathrm{~km}$, Guaranda a 146 km y el Tena a 227 km; para Guayaquil hay una distancia de $335 \mathrm{~km}$.

\subsubsection{Condiciones demográficas}

La población de la provincia de Chimborazo tiene un total de 458.583 habitantes, lo que corresponde al 3,17\% de la población total en Ecuador, según datos del INEC para el año 2010. 


\subsubsection{División político administrativa}

La provincia de Chimborazo está dividida en 10 cantones: Riobamba (capital), Cumandá, Alausí, Colta, Chambo, Chunchi, Guamote, Guano, Pallatanga, y Penipe.

\subsubsection{Clima}

Las temperaturas oscilan entre los $6 \mathrm{C}$ a los $20^{\circ} \mathrm{C}$, con una promedio de $13^{\circ} \mathrm{C}$.

\subsubsection{Orografía}

Esta provincia contempla una serie de cadenas montañosas que unen los ramales principales de la Cordillera de los Andes. Destaca el nudo de Tiocajas, de las hoyas de Chambo y Chanchán. Una de las elevaciones más importantes es el Chimborazo [2].

\subsubsection{Hidrografía}

El principal sistema hidrográfico lo constituyen el río Chambo y sus afluentes del Guamote, Chibunga, Guano, Sicalpa, San Juan y Blanco. Al Sur está la hoya del río Chanchán y sus afluentes son los ríos Guasuntos, Sibambe y Chuchi desembocan al Chimbo [2].

\subsection{Visión económica productiva de Chimborazo}

Impulsar sistemas de producción sostenible y procesos asociativos en cadenas productivas y de valor, emprendimientos competitivos y complementarios mediante una comercialización justa y equitativa que favorezca la producción e industrialización agrícola, pecuaria, artesanal y turística con clara identidad cultural e histórica asociada a un estilo de desarrollo sostenible y solidario fortaleciendo capacidades locales recuperando y optimizando los recursos naturales y culturales.

En el marco del PDOT se plantea la provincia de Chimborazo lograr un fortalecimiento del sector primario y un sector secundario que cuente con productos innovadores, patrimoniales y de impacto social con alto valor agregado y con un mercado que permite la comercialización justa, aportando al fomento de la soberanía alimentaria de los chimboracenses.

Además se espera lograr por lo menos el $4 \%$ del PIB Nacional, actualmente el aporte provincial es del $1.67 \%$ del nacional. Para ello se pretende cambiar la distribución de la PEA entre los sectores de la producción primario y secundario, al $70 \%$ y $30 \%$ 
respectivamente. Actualmente entre ambos sectores están el $80 \%$ en la producción primaria y el $20 \%$ en el sector secundario (o de valor agregado).

El Gobierno de la Provincia cuenta con un Sistema de Fomento Productivo que constituye un mecanismo práctico que busca por un lado la articulación entre las políticas nacionales, estrategias provinciales, cantonales y parroquiales (participación ciudadana, organización territorial, economía popular y solidaria, soberanía alimentaria entre otros) en torno a la gestión concertada del sistema económico y productivo sostenible del territorio, en coherencia con los procesos de planificación y ordenamiento territorial buscando como uno de los fines alcanzar mayor equidad en algunos casos y otros una competitividad de tipo incluyente.

Al ser la actividad económica de Chimborazo principalmente productiva, es necesario dar un salto de la producción primaria a la innovación y valor agregado, debido a que si continúa siendo el sector primario el que involucra a la mayoría de la PEA, los cambios que se pretenden en la visión provincial difícilmente se darán. Sin embargo ante la situación de la Provincia es indispensable la creación de unas condiciones favorables para que este salto de sector económico sea efectivo y sostenible:

1. Construcción de una identidad productiva.

2. Creación del entorno favorable del apoyo a la producción (Infraestructura).

3. Fortalecimiento del tejido social vinculado a las actividades productivas.

4. Desarrollo de un entorno normativo y regulatorio favorable y amigable al fomento productivo.

5. Gestión de la información básica productiva confiable y actualizada.

6. Investigación de mercados.

\section{Desarrollo y Discusión}

\subsection{La globalización un punto de partida}

Globalización es un término que remite a fenómenos que adquieren un carácter global, es decir, mundial, planetario, rebasando (y subyugando) las fronteras y las identidades locales, las economías y los sistemas políticos [3].

Debe diferenciarse la 'globalización' como tendencia al acercamiento de las sociedades humanas entre sí, de un campo de lucha entre orientaciones culturales antagónicas. Este acortamiento de las distancias, aceleración de los tiempos, configuración de un solo planeta que opera en una sociedad determinada, que habitualmente es la sociedad capitalista con sus formas económicas y políticas específicas, donde 
marca el dominio de las finanzas sobre la producción, por ello la globalización debe ser entendida como la construcción del mundo bajo el dominio exclusivo del gran capital transnacional 'globalizado', que modifica la economía y la política, los mercados y los estados, de acuerdo a sus necesidades de acumulación de ganancias.

En este sentido, la globalización es una tendencia propia del desarrollo del capitalismo como economía - mundo, que se aceleró con las nuevas tecnologías que, a su vez, aceleran los flujos de capitales y mercancías. Algunas de las características de la globalización es la integración global de la economía con el dominio de las fuerzas del Mercado, la transformación de los sistemas productivos y de los mercados de trabajo, velocidad del cambio tecnológico y revolución de los medios de comunicación de masas y expansión del consumismo. La globalización ocurre en la producción, en el conocimiento y en las finanzas.

La globalización de los mercados internacionales registrada en los últimos años, representa un reto para los países, de economías en desarrollo y, dentro de éstos, para los productores a pequeña y mediana escala. Ante las limitaciones de factores de producción que disponen y su bajo poder de negociación en el mercado, este tipo de productores sienten la necesidad de mejorar las condiciones que los ubican en condiciones de pobreza, mediante la adopción de prácticas productivas y comerciales, alternativas e innovadoras, que den adecuada respuesta a los requerimientos de las nuevas tendencias de consumo.

Actualmente, los acelerados procesos de globalización obligan a los Estados a asumir un rol más activo en su modo de interrelacionarse con los diferentes actores de la nueva economía mundial del poder. En el caso de nuestro país, a partir del Plan Del Buen Vivir se recoge que, la inserción estratégica y soberana se propicia en un escenario nuevo que rompe esa lógica del sistema neoliberal ha hecho que el mercado sea el eje regulador de las relaciones internacionales, al contrario se da un énfasis en el cambio hacia la economía endógena para el Buen Vivir, que a su vez pretende el fomentar las relaciones comerciales y de cooperación internacional con gobiernos autónomos descentralizados a lo largo del mundo [4].

No hay duda de que la globalización ha traído significativos beneficios al consumidor norteamericano. Ha reducido los precios de bienes antes considerados de lujo, como televisores de pantalla grande y duraznos en invierno; además ha incrementado el poder de compra de los ciudadanos de bajos recursos. Ha contribuido a controlar la inflación y ha fomentado que millones de americanos inviertan sus recursos en acciones, ampliando los mercados de bienes y servicios. Ha posibilitado también que pueblos como China e India reduzcan dramáticamente la pobreza y ha consolidado los mercados a largo plazo para permitir un mundo más estable.

Pero tampoco se puede negar que la globalización ha incrementado fuertemente la inestabilidad de millones de americanos de menores recursos. Para mantenerse 
competitivas y lograr que los inversionistas estén contentos en el mercado global, las compañías establecidas en Estados Unidos se han automatizado, disminuido su tamaño y establecido en otros países. Han enfatizado los operativos para obtener mayores utilidades y han restado importancia a los planes de jubilación y programas de salud, lo que implica mayores gastos y riesgos para los trabajadores.

\subsection{Apuestas nacionales frente a la producción}

Según SENPLADES en el plan del Buen vivir, entre las grandes apuestas nacionales está el impulso a la industria nacional de alimentos, asegurando la recuperación y la innovación de productos de calidad, sanos y de alto valor nutritivo, articulando la producción agropecuaria con el consumo local. Sin perder de vista que busca fomentar la producción de alimentos sanos y culturalmente apropiados para el consumo nacional, evitando la dependencia de las importaciones.

En este sentido la productividad, la diversificación y la generación de valor agregado son caminos necesarios y urgentes previstos en la planificación del país. La productividad territorial requiere de sinergias entre los diferentes sistemas productivos, tanto rurales como de los grupos urbanos, para que se perfilen estrategias de una economía endógena. Se busca una productividad sistémica por encima de la competitividad, en el sentido de servir mejor a un modelo de desarrollo que privilegie las condiciones de vida, las capacidades y potencialidades humanas; y no se piense desde las posibilidades de procesos de globalización. Es por ello que en el Código de la Producción [5] se recoge el Desarrollo Territorial Equitativo expresado en los artículos:

Art. 69: Priorización territorial. La transformación productiva buscará dinamizar todos los territorios del país; no obstante, se priorizará la inversión pública en desarrollo productivo en las zonas económicamente deprimidas, tomando en cuenta factores como: Altos índices de desempleo, necesidades básicas insatisfechas, entre otros; los mismos que serán determinados conjuntamente con la Secretaría Nacional de Planificación, el Consejo Sectorial de la Producción y los Gobiernos Autónomos Descentralizados. Estos organismos evaluarán y monitorearán el cumplimiento de esta política.

Art. 70: La Secretaría Nacional de Planificación, el Consejo Sectorial de la Producción y los Gobiernos Autónomos Descentralizados podrán definir políticas para impulsar mecanismos de promoción del desarrollo económico endógeno de los territorios, y de integración con el mercado nacional e internacional.

Existe un planteamiento de superar la dicotomía entre lo urbano-rural, en que la producción agrícola entra en el sistema nacional e internacional, sin que exista una relación de consumo hacia los nodos urbanos más cercanos, y en la que los centros urbanos ofrecen servicios y productos que tienen poca relación con la producción 
material y humana local, es necesario: La conformación o fortalecimiento de la estructura poli céntrica, desde una perspectiva de cadena de valor y generación de empleo, que implica fortalecer los nodos de sustento (agro)productivo, correspondientes a los ámbitos básicos del desarrollo rural: luego, vincular estos nodos a la productividad sistémica. Entre las tipologías el grupo de vinculación regional se debe especializar en procedimientos primarios (industrias o manufacturas de primer orden, procedimientos de uno o dos productos primarios); los grupos de estructuración nacional se deben enfocar hacia procedimientos avanzados (industrias especializadas donde confluyen varios productos primarios); y los grupos de articulación internacional se deben orientar en procedimientos especializados (industria de (alta) tecnología, especialización científica).

Por otra parte se busca la diversificación de la producción agroalimentaria: Soberana, sana, eficiente, donde la producción primaria o rural deberá considerar tres principales mercados, en el siguiente orden:

1. El consumo interno que permita garantizar la soberanía alimentaria;

2. La producción para la industria nacional con miras a encadenamientos productivos, generación de empleo y valor agregado a la producción; y

3. La exportación, considerando la inserción inteligente y soberana en el mundo mediante la ubicación de mercados alternativos de productos y destinos.

La distribución actual de la producción primaria en el país es muy heterogénea; ciertas áreas se dedican casi exclusivamente a productos agrícolas de exportación, mientras otras a la producción de alimentos para el consumo interno. En general hay una producción deficiente para el desarrollo industrial o manufacturero. Diversificar la producción es un eje importante para establecer los ámbitos de sustento al desarrollo rural, en particular para asegurar las cadenas cortas y el retorno de los flujos de riqueza a los espacios rurales.

La misma diversificación introduce otros conceptos de eficiencia que van más allá de la producción agrícola misma, incorporando todo el ciclo de los productos hasta el consumo, porque reduce costos e impactos de transacciones y de transporte. Finalmente, contribuye a un desarrollo sustentable, porque un espacio de producción diversificada desde el punto de vista biológico, en contraste con grandes extensiones de monocultivos, ocupa menos fertilizantes o pesticidas, y puede fácilmente controlar sus cultivos en base a prácticas agroecológicas [6]. En el Código de la Producción se establece la Eco-eficiencia y Producción Sostenible.

Art. 232: Se entenderán como procesos productivos eficientes el uso de tecnologías ambientalmente limpias y de energías alternativas no contaminantes y de bajo impacto; adoptadas para reducir los efectos negativos y los daños en la salud de los seres 
humanos y del medio ambiente. Estas medidas comprenderán aquellas cuyo diseño e implementación permitan mejorar la producción, considerando el ciclo de vida de los productos así como el uso sustentable de los recursos naturales. También, se entenderán como procesos productivos más eficientes y competitivos, la implementación de tecnologías de punta, que permitan mejorar la administración y utilización racional de los recursos, así como prevención y control de la contaminación ambiental, producto de los procesos productivos, la provisión de servicios y el uso final de los productos.

El reconocimiento de la función social y ambiental de la propiedad, el incentivo a la agroecología y la diversificación productiva, deben complementarse con la identificación y planificación de los requerimientos de abastecimiento nacional de alimentos, en consulta con productores y consumidores.

Adicionalmente, desde los territorios rurales se generan las condiciones de base para la soberanía alimentaria. La misma que se sustenta en el reconocimiento del derecho a la alimentación sana, nutritiva y culturalmente apropiada, para lo cual es necesario incidir tanto en las condiciones de producción, distribución y consumo de alimentos.

El mejoramiento de la productividad rural requiere además de centros de apoyo técnico y capacitación, así como de la promoción de centros de investigación y desarrollo aplicados a la producción agropecuaria de acuerdo a las potencialidades existentes.

En términos de planificación territorial, la zonificación del uso del suelo agrícola debe realizarse en función de la aptitud del mismo, considerando vocaciones productivas existentes, y deberá velarse por el cumplimiento en el uso establecido. También se deberán incorporar mecanismos para la democratización de los medios de producción (tierras, agua, créditos y mercados) así como gestionar sistemas de almacenamiento, de riego y de movilidad, adecuados para fomentar la producción.

\subsection{El comercio como base del mercado}

Se denomina comercio a la actividad socioeconómica consistente en el intercambio de algunos materiales que sean libres en el mercado de compra y venta de bienes y servicios, sea para su uso, para su venta o su transformación. Es el cambio o transacción de algo a cambio de otra cosa de igual valor [7].

En un marco de buscar una adaptación al comercio para la provincia de Chimborazo se ha identificado tres tipos de comercio responsable (comercio equitativo, comercio 'verde' y, más recientemente, comercio ético) se han desarrollado separadamente a menudo en competencia entre sí para atraer la atención del consumidor, pero en la actualidad están comenzando a converger en importantes aspectos.

El comercio equitativo ha sido definido por sus seguidores como: 
una asociación comercial, basada en el diálogo, la transparencia y el respeto, que procura que haya mayor equidad en el comercio internacional. Contribuye al desarrollo sostenible al ofrecer mejores condiciones comerciales para trabajadores y productores marginados, especialmente del Sur, y para asegurar los derechos de unos y otros.

El comercio 'verde' significa que se usan productos medioambientales y normas de fabricación que regulan el impacto medioambiental de los productos que se hallen en producción, en activo o en fase de eliminación, tanto si provienen de fabricantes y productores como si han sido reclamados por los compradores en la cadena de suministro. A menudo el comercio 'verde' está asociado con alguna forma de 'etiqueta ecológica'.

El comercio ético es el enfoque seguido por las marcas principales y los minoristas para conseguir que sus cadenas de suministro estén libres de problemas que dañan la reputación, como, por ejemplo, el abuso de los derechos humanos y el trabajo infantil [8].

Su finalidad es lograr que las instalaciones de producción de las cadenas de suministro respeten las normas básicas de los derechos humanos en el lugar de trabajo, protejan la salud y la seguridad, y eviten el empleo de niños o los trabajos forzados. En razón que las características de los productores de Chimborazo son de pequeñas unidades familiares, la opción factible en la misma identificación de un comercio responsable se identifica el comercio justo.

Según Andrade [9] El Comercio Justo, es una relación comercial a largo plazo, basada en el diálogo, la transparencia y el respeto, que conlleva al logro de una mayor justicia en el marco del comercio internacional; y, más allá de los criterios económicos, el comercio justo tiene en cuenta valores éticos que abarcan aspectos sociales y ambientales.

El Comercio Justo o Economía Solidaria: Se define como una solución alternativa al comercio internacional tradicional. Se trata de una asociación comercial que favorece el desarrollo sostenible de los productores excluidos o desfavorecidos garantizando mejores condiciones comerciales, aumentando la sensibilización realizando campañas al efecto. Los criterios que deben cumplir los productos de este tipo de comercio varían según el producto, pero incluyen aspectos tales como la garantía de precios, el pago previo y el pago directo a los productores y sus cooperativas. En definitiva, el Comercio Justo implica el precio justo, es decir, que éste no contenga componentes influenciados por intervenciones o subsidios estatales.

El sistema Comercio Justo (FT, por Fair Trade) o Comercio Alternativo (AT, por Alternative Trade) es una iniciativa para crear canales comerciales innovadores, dentro de los cuales la relación entre partes se orienta al logro del desarrollo sustentable y sostenible de la oferta. El FT se orienta hacia el desarrollo integral, con sustentabilidad 
económica, social y ambiental, respetando la idiosincrasia de los pueblos, sus culturas, sus tradiciones y los derechos humanos básicos [10].

La filosofía del Comercio Justo o Economía Solidaria consiste en: Que la mejor ayuda de los países desarrollados a los países en desarrollo es el establecimiento de relaciones comerciales éticas y respetuosas, con crecimiento sostenible de las naciones y de los individuos; más que por las entidades oficiales o estatales.

Los principios del Comercio Justo son:

1. Los productores forman parte de cooperativas u organizaciones voluntarias y funcionan democráticamente.

2. Libre iniciativa y trabajo, en rechazo a los subsidios y ayudas asistenciales (de allí la frase del comercio justo: «Comercio, no ayuda»).

3. Rechazo a la explotación infantil.

4. Igualdad entre hombres y mujeres.

5. Se trabaja con dignidad respetando los derechos humanos.

6. El precio que se paga a los productores permite condiciones de vida dignas.

7. Los compradores generalmente pagan por adelantado para evitar que los productores busquen otras formas de financiarse.

8. Se valora la calidad y la producción sustentable.

9. Cuidar del medio ambiente.

10. Se busca la manera de evitar intermediarios entre productores y consumidores.

11. Se informa a los consumidores acerca del origen del producto.

12. El proceso debe ser voluntario, tanto la relación entre productores, distribuidores y consumidores.

Competitividad como opción de mejoramiento: Entendemos por competitividad a la capacidad de una organización pública o privada, lucrativa o no, de mantener sistemáticamente ventajas comparativas que le permitan alcanzar, sostener y mejorar una determinada posición en el entorno socioeconómico. La competitividad no es producto de una casualidad ni surge espontáneamente; se crea y se logra a través de un largo proceso de aprendizaje y negociación por grupos colectivos representativos que configuran la dinámica de conducta organizativa, como los accionistas, directivos, empleados, acreedores, clientes, por la competencia y el mercado, y por último, el gobierno y la sociedad en general [11]. 
Competitividad sistémica se define como el proceso de construcción de ventajas competitivas dinámicas, basadas en la existencia de competitividad social, económica, ambiental y de inserción externa. Esta contempla transversalmente las potencialidades de los recursos naturales, pero también las restricciones que ellos imponen a la actividad económica, con una visión de justicia intergeneracional [12].

La competitividad como un concepto comparativo fundamentado en la capacidad dinámica que tiene una cadena agroalimentaria, un cluster industrial, o actividades artesanales, turísticas, de servicios o económico productiva en general, localizada espacialmente, para mantener, ampliar, y mejorar de manera continua sostenida su participación en el Mercado, tanto domestico como internacional, a través de la producción, distribución, y venta de servicios en el tiempo, lugar y forma solicitados, buscando como fin último el beneficio de la sociedad [13].

La constitución establece la prioridad de buscar una competitividad sistémica basada en un modelo que incluya por un lado el capital empresarial y laboral, el capital organizacional e intelectual y el capital macroeconómico y, por otro, el capital comercial, institucional y gubernamental y el social de confianza, lo cual es rol de todas las instituciones que hacen parte del sistema económico nacional. Pero el manejo de la competitividad debe estar complementado con políticas que permitan un desarrollo equilibrado del territorio y aseguren una adecuada distribución de ingresos y de la riqueza nacional.

El desarrollo competitivo de los territorios en una perspectiva pobre y contra sesgos étnicos y de género. Implica además de un contexto macro favorable que genera certidumbre en los empresarios y sus inversiones, un conjunto de elementos de política y de inversiones a nivel de los territorios, que en conjunto y en forma sistemática promueven el desarrollo competitivo.

1. Cinco campos de acción para promover la competitividad sistemática territorial;

2. Un marco adecuado de políticas e instituciones, tanto a nivel nacional como territorial;

3. Unas políticas sociales y programas dirigidos a incrementar los activos de las familias pobres;

4. Un programa dirigido a mejorar las competitividad de las empresas;

5. Un programa dirigido a mejorar los ingresos y empleos de los pobres

6. Un sistema institucional para el desarrollo territorial.

La innovación y la calidad elementos claves: Las micro, pequeñas y medianas empresas (MIPYMES) tienen dificultades para conocer las condiciones en las que se desarrollan las actividades comerciales en los mercados, como consecuencia de las 
limitaciones de recursos técnicos y financieros. Sus condiciones son insuficientes para solventar las mejoras técnicas y de calidad requeridas en sus productos o servicios para mantenerse en los mercados.

La innovación agraria es un proceso construido socialmente. La innovación es el resultado de la interacción de una gran cantidad de agentes y partes interesadas. Si la investigación y extensión agrícola son importantes para la innovación agraria, también lo son los mercados, los sistemas gubernamentales, las normas sociales y, en general, una gran cantidad de factores que crean los incentivos para que un agricultor decida cambiar la manera en la que trabaja, y que premie o bloquee sus decisiones [14].

El efecto indirecto de la innovación agrícola - principalmente a través de los precios más bajos de los alimentos - es una contribución fundamental para aumentar el bienestar de consumidores de escasos recursos que son compradores netos de alimentos; y también de los productores.

Las políticas e intervenciones públicas deberían tener la meta de explotar los enlaces entre los bienes públicos y los intereses privados. Las políticas específicas que podrían llevar a este escenario en que todos ganan, incluyen: La protección de estos sistemas agrícolas contra la competencia comercial injusta y las barreras no arancelarias para el comercio; la promoción de la cooperación privada-pública; el desarrollo de las políticas nacionales de ciencia y tecnología que no discriminen a favor de ningún tipo particular de organización de investigación; el apoyo de la investigación básica y estratégica; la financiación de la formación y capacitación continua de los científicos; la promoción y establecimiento de acuerdos de cooperación entre universidades nacionales e institutos de investigación y sus contrapartes en otros países; el estímulo de lazos más fuertes y cooperación más explícita entre diferentes actores dentro de las cadenas productivas y de mercadeo.

Abordar un marco de sistemas de innovación es tener en buena medida las oportunidades y necesidades de innovación, especialmente cuando un rápido cambio está en curso. El contexto incluye las condiciones de política, mercadeo y comercio y los desafíos que éstos presentan, así como otros factores del contexto, como el ambiente sociopolítico y la base de recursos naturales [15]. Los temas principales:

1. Actores, las funciones que desempeñan y las actividades en las cuales están involucrados, con énfasis en la diversidad de actores de los sectores público y privado y en lo apropiado de sus papeles.

2. Actitudes y prácticas de los principales actores, con énfasis en la colaboración, potenciales ineficiencias, patrones de confianza y la existencia de una cultura de innovación.

3. Patrones de interacción, con énfasis en redes y asociaciones, inclusión y la existencia y funciones de organismos potenciales de coordinación y de los beneficiarios. 
4. Ambiente facilitador (políticas e infraestructura), con énfasis en el papel

de las políticas relacionadas con ciencia, tecnología y preocupaciones fiscales; el papel de las organizaciones de agricultores y otras organizaciones en la definición de los desafíos a la investigación de la innovación, y la significación de los marcos legales.

Las principales características de la innovación se han identificado los siguientes:

1. La innovación no es ni ciencia ni tecnología sino la aplicación de conocimiento de todo tipo para alcanzar resultados sociales y económicos deseados.

2. Con frecuencia la innovación combina cambios técnicos, organizacionales y de otro tipo.

3. La innovación es el proceso a través del cual las organizaciones 'dominan e implementan el diseño y producción de bienes y servicios que son nuevos para ellas, independientemente de si son nuevos para sus competidores, su país o el mundo' [16].

4. La innovación comprende mejoramientos radicales y muchos de pequeña escala y un proceso continuo de actualización.

5. La innovación puede ser provocada de diferentes maneras.

6. Un considerable nivel de valor está siendo agregado en los sectores agrícolas no tradicionales.

Los patrones de interacción necesarios para crear sistemas dinámicos de innovación con frecuencia no se encuentran presentes. Con demasiada frecuencia, agricultores, empresarios y empresas no son parte de las redes de organizaciones de investigación, capacitación y desarrollo, requeridas para llevar a término un proceso continuo de innovación.

La resistencia a formar tal tipo de relaciones se refuerza por patrones de comportamiento arraigados y por la desconfianza, la que se origina en los papeles que esas organizaciones han jugado en ambientes previos, menos dinámicos y desafiantes económicamente.

La evidencia de que las actitudes y prácticas son una dificultad, proviene del hecho de que fuertes incentivos para innovar, que surgen de la exposición a mercados altamente competitivos, rara vez han sido suficientes para inducir nuevos patrones de colaboración. Esta carencia de interacción tiene varias consecuencias:

1. Limitado acceso al conocimiento. Los agricultores, microempresarios y empresas no tienen acceso a las fuentes de conocimiento que necesitan para solucionar sus problemas, crear nuevos productos y procesos y, por tanto, lidiar con los problemas y competir. 
2. Débil articulación de la demanda por investigación y capacitación. Las políticas, currículo de capacitación y esfuerzo de investigación por parte de los organismos públicos están desconectados de las necesidades y de los intereses del sector.

3. Aprendizaje tecnológico débil o ausente. Las oportunidades para dominar nuevos conocimientos a través de la colaboración con otros son limitadas.

4. Debilidad o carencia de aprendizaje institucional a nivel de la empresa, agricultor, empresario o sectorial. Sólo existen oportunidades restringidas de construir conocimiento acerca de la forma como se debe innovar en respuesta a condiciones rápidamente cambiantes.

5. Débil actualización del sector. Las organizaciones no son efectivas para enfrentar los estándares comerciales cambiantes o para desarrollar una imagen de marca nacional.

6. Débil integración de las preocupaciones sociales y ambientales en la planeación y desarrollo del sector. El sector está usualmente preocupado con la producción y las ganancias pero presta poca atención a las condiciones ambientales y sociales en las cuales opera. Esta actitud puede crear problemas ambientales y tensión social y reducir su acceso a los mercados de exportación.

7. Débil conexión con fuentes de financiamiento para la innovación. La innovación no se refiere únicamente a nuevo conocimiento o nuevas prácticas sino también a la inversión en la capacidad para aplicar las novedades en gran escala. El proceso de innovación puede fracasar debido a que no existen los medios financieros para introducir el cambio a gran escala (Tabla 1).

\section{Table 1}

Incentivar la innovación.

\section{Liderada por oportunidades}

Fase de surgimiento

Fase de estancamiento

Fase de un sistema dinámico de innovación

Fuente: Banco Mundial (2006).

\section{Dirigida}

Fase de expansión

Fase de sistema dinámico de innovación

La innovación orientada al mercado, si bien puede implicar inversiones y costos, tiene la ventaja de que reviste mayor seguridad sobre los beneficios (precio, certeza de venta) y menores riesgos. Las ideas de innovación pueden surgir en los propios trabajadores del campo, aunque esto resulta poco común, pues las zonas rurales suele existir un referente muy limitado del entorno. En la mayoría de casos las innovaciones han sido propuestas desde personas externas (agentes externos): El punto clave para es 
la capacidad para recuperar las ideas de innovación, adoptarlas y ponerlas en práctica (Tabla 2).

Los tipos de innovación orientada al mercado que se desarrollan con más frecuencia son:

1. Innovación tecnológica;

2. Innovación en procesos comerciales;

3. Innovación en procesos gerenciales;

4. Innovación en aspectos financieros;

5. Innovación en servicios y logística.

Table 2

Innovación acorde a los grupos de productores.

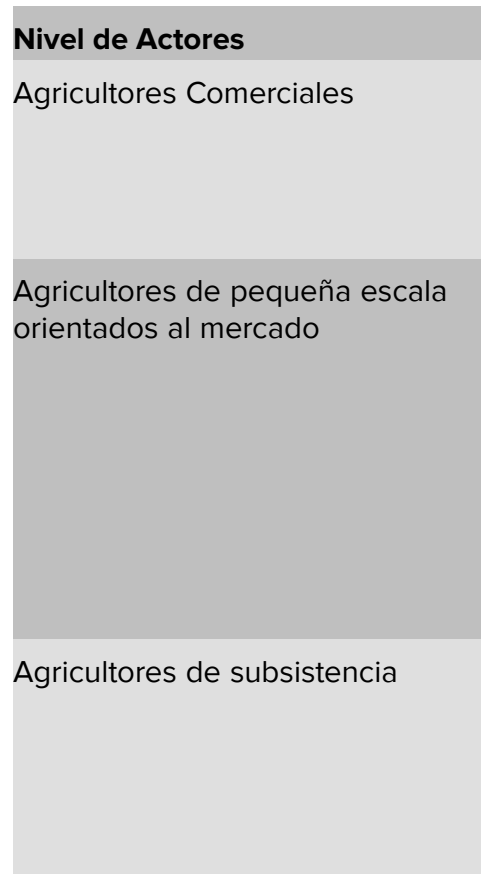

Fuente: Banco Mundial (2006).

Tipo de Sistema
Sistema intensivos de producción
para alimentos básicos no
transables
-Sectores de alto valor
- Diversificación de sistema de
producción
-Producción intensiva de
productos básicos para dejar
tierra disponible para productos
de alto valor
-Sistemas de producción de
productos de alto valor y bajo
volumen
-Sistema de producción de
alimentos básicos
-Construcción de capital humano
y social para abordar un conjunto
de oportunidades de generación
de ingresos

\section{Tipo de Sistema} para alimentos básicos no transables

-Sectores de alto valo - Diversificación de sistema de productos básicos para dejar de alto valor productos de alto valor y bajo - Sistema de producción de alimentos básicos y social para abordar un conjunto de ingresos

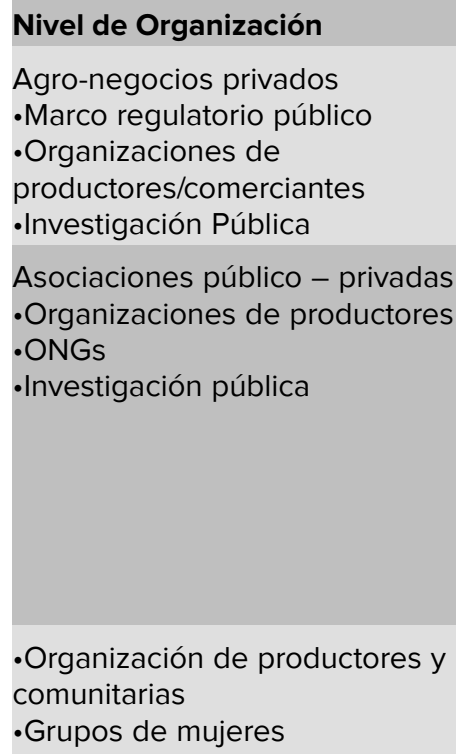

\section{Nivel de Organización} - Investigación Pública

Asociaciones público - privadas - Organizaciones de productores - ONGs

- Investigación pública

La innovación no se limita solo al producto, sino también en la información del mercado, en la logística de entrega, en la garantía de una marca y en otros factores valorados por el cliente. Las unidades productivas exitosas han desarrollado el concepto integral de calidad como estrategia de competitividad. Esto quiere decir que la calidad no se ha limitado únicamente al producto sino que está presente en todos los elementos relacionados con la satisfacción del cliente, es decir: Calidad en el producto, en el proceso de producción y de agregación de valor, en los servicios que acompañan el producto, en el mercadeo y en la gerencia de la empresa. 
1. Innovar con enfoque de mercado.

2. Adoptar ideas de innovación.

3. Mejorar la calidad.

Enfoque de cadena productiva para innovar: La cadena productiva es un sistema constituido por actores interrelacionados y por una sucesión de operaciones de producción, transformación y comercialización de un producto o grupo de productos en un entorno determinado.

El enfoque de cadena es pertinente en el contexto actual de evolución de la economía mundial, competitividad, globalización, innovación tecnológica y complejos sistemas agroalimentarios. Este enfoque nos permite dar una mirada sistemática a las actividades productivas.

Una cadena productiva puede ser competitiva o no. Una cadena productiva es competitiva cuando sus actores han desarrollado capacidades y condiciones para mantenerse y aumentar su participación en el mercado de manera sostenible. La Competitividad de una cadena tiene que ver con las habilidades, actitudes, talentos, competencias, visión empresarial de sus actores. Algunos autores hablan de competitividad sistémica para hacer entender que una cadena es competitiva no solo cuando una empresa o un grupo de actores tienen rentabilidad, sino que el conjunto de actores es competitivo. Gestión de calidad es gestión de la cadena productiva y su consecuente cadena de valor.

\subsection{La experiencia Ecuatoriana en innovación y exportación}

Auge del Cacao en el Ecuador: Gran parte de la historia económica del Ecuador tiene que ver con la producción y exportación de cacao. Este producto, conocido en el país con el nombre de la 'pepa de oro', sirvió para que el Ecuador se incorpore al mercado mundial y además el cacao fue un nervio central de la economía ecuatoriana, favorecido por la demanda de los países industrializados que tenían crecientes excedentes para endulzar sus paladares, por la nueva tecnología en la construcción de barcos que bajó los precios de la transportación, las excepcionales condiciones naturales del puerto de Guayaquil y su sistema fluvial, el clima propicio para el crecimiento de la fruta y las condiciones internas de producción. La producción del cacao se realizó en enormes latifundios, que se crearon violentamente a través del despojo a propietarios.

En un inicio, el cacao surgió en el Ecuador de manera silvestre y fue propagado por todo el litoral gracias a los abundantes simios por todo el trópico y subtrópico de la Costa nacional. Para finales de la Colonia, Guayaquil tenía fama de producir el mejor cacao del mundo y fue, precisamente, la riqueza generada por su exportación, la que 
proveyó de los recursos para la Independencia de la Audiencia de Quito y su posterior conversión en República del Ecuador.

Sin duda, las exportaciones de cacao se convirtieron, con el paso del tiempo, en la mayor fuente de ingresos de divisas para el Ecuador hasta la mitad del siglo XX. En efecto, entre 1830 y 1950, solo durante 9 años las exportaciones de cacao no lideraron las ventas externas del país y, para 1875, nuestro país era el principal exportador de cacao del mundo.

Un balance objetivo de los cambios económicos, sociales y políticos que produjo el boom cacaotero, entre 1875 y 1925, nos muestra que se creó un modelo de desarrollo basado en la exportación de productos agrícolas no procesados y la importación de productos manufacturados, con lo cual se creó un modelo de desarrollo dependiente a nombre del discurso liberal del libre comercio.

El cacao ecuatoriano se vino a menos cuando los ingleses se llevaron cepas de los árboles ecuatorianos y los cultivaron en sus colonias de la costa occidental del África, con lo que surgió una competencia que debilitó la producción nacional, pues la cercanía de la mencionada costa africana de Europa le permitía llegar con mayor agilidad y mejores precios al mercado procesador del cacao.

Adicionalmente, con la finalidad de acelerar el tiempo entre la siembra y la cosecha, los productores ecuatorianos introdujeron una variedad venezolana de cacao hacia finales del siglo XIX. Lamentablemente dicha introducción trajo consigo plagas, como la monilla y la escoba de bruja, lo que diezmó la producción cacaotera nacional hasta cerca del colapso. Los únicos árboles que resistieron fueron los de río arriba, esto es los del subtrópico en las estribaciones de los Andes, donde no llegaron las plagas por las barreras geográficas y porque en esa zona no se sembraron los árboles venezolanos contaminados.

Precisamente son esos árboles milenarios los que actualmente producen el famoso cacao de aroma conocido como de río arriba, que sirve de materia prima para la elaboración de los más exquisitos y famosos chocolates del mundo. Pero, lamentablemente, al igual que en épocas coloniales, en la actualidad el Ecuador sigue produciendo y exportando 'pepas' de cacao, sin ningún valor agregado, para que el primer mundo produzca el mejor chocolate de la tierra. Suiza, Francia, Italia, entre otros, importan el cacao ecuatoriano para la producción de sus famosos 'bombones' que satisfacen la demanda de sus mercados internos y les significan un importante rubro de exportación al resto del mundo.

Para el Ecuador el auge cacaotero no logró crear los suficientes eslabonamientos económicos para arrastrar a las demás regiones. Se mantuvo una dualidad en el agro ecuatoriano: Una economía de exportación en la Costa y el sistema de hacienda tradicional en la Sierra. La cordillera andina resultó un enorme obstáculo para la articulación de la Sierra, cuestión que se remarcó por la vigencia de una economía tradicional que 
no podía ofertar los productos que demandaba la Costa, que debió importar incluso bienes de subsistencia. Es decir, se produjo el crecimiento de una sola región, mientras se mantenía la economía rezagada de las demás, lo cual más bien introdujo nuevas diferencias y distorsiones para un crecimiento más equitativo y homogéneo.

Auge Bananero en el Ecuador: La crisis económica iniciada en 1925, que llegó a su clímax en 1931, se prolongará hasta 1939, en medio de una serie de devaluaciones que buscaban hacer competitivas las exportaciones y de un conjunto de políticas monetarias poco profundas que no tuvieron la capacidad de introducir reformas estructurales. Mas bien, un nuevo acontecimiento, la Segunda Guerra Mundial incrementará las exportaciones y para fines de la década del 40 , un nuevo producto de exportación, el banano, iniciará un nuevo boom agro - exportador ahogando los tímidos intentos de poner en marcha un nuevo modelo de industrialización por sustitución de importaciones, que otros países latinoamericanos habían emprendido.

Los países en guerra aumentaron la demanda de arroz, balsa, caucho, cascarilla, cacao y café lo cual permitió una triplicación de las exportaciones entre 1939 y 1945. Adicionalmente, la balanza comercial desfavorable que el país tuvo hasta 1940 comenzó a cambiar porque creció la demanda de Estados Unidos que entró a la guerra y bajaron las importaciones por problemas de transporte. La Segunda Guerra Mundial favoreció la recuperación de la economía ecuatoriana: Subió el precio de los productos de exportación, aumentó la demanda de nuevos productos hacia Estados Unidos, y reingresaron capitales nacionales y extranjeros por la mayor seguridad que les ofrecía un país no involucrado en la guerra. Sin embargo, el crecimiento del circulante produjo una inflación de tres veces entre 1939 y 1944.

Entre 1946 y 1948 las exportaciones de café y cacao volvieron a incrementarse, respondiendo al ciclo de expansión del capitalismo mundial. A partir de 1948 comenzó el impetuoso crecimiento de la exportación bananera favorecido por las plagas, huracanes y ciclones que impactaron en las plantaciones centroamericanas [17]. El precio del banano y las exportaciones subieron hasta 1964, época en la que se produjo su rápida declinación. Este nuevo ciclo de agro - exportaciones mostrará cuatro diferencias importantes respecto al período cacaotero.

Primero, fue el estado el que estimuló las inversiones bananeras a través de créditos, subsidios, entrega de tierra y devaluaciones monetarias para mejorar su competitividad y las ganancias de los productores y exportadores.

Segundo, se originó una nueva ‘burguesía agraria’ de pequeños y medianos productores, integrada por antiguos propietarios, comerciantes y profesionales que abrieron agresivamente nueva frontera agrícola hacia las estribaciones de la cordillera, para controlar el $80 \%$ de la producción, en tanto el otro $20 \%$ estuvo a cargo de las grandes plantaciones [18]. 
Tercero, las relaciones sociales que establecieron las diversas unidades de producción fueron definitivamente salariales, es decir, se superó la ambigüedad de las relaciones en 'transición' que caracterizaron a la época cacaotera, y enviaron un importante mensaje a la hacienda serrana, sobre todo a la tradicional, que aún mantenía las formas serviles tipo 'huasipungo' en el campo.

Cuarto, sin embargo de estas importantes matizaciones introducidas al modelo agro exportador, se mantuvo e incluso acentuó la dependencia, tanto porque el país continuó su papel de exportador de materias primas e importador de bienes de capital, como porque, las grandes compañías transnacionales controlaron la comercialización, que manejaban el 80\% de las exportaciones en 1954 e incluso subieron al 90\% en 1964 [19]. De manera marginal, algunas empresas ecuatorianas, algunas muy pequeñas, lograron incorporarse en la comercialización.

Las haciendas serranas y la salida hacia la productividad: Las haciendas instrumentaron varias tácticas tradicionales para intentar revertir la crisis: La ampliación de la frontera agrícola hacia las alturas de los páramos desmontando el matorral virgen, actividad que delegó a una serie de partidarios (aparceros), a quienes les entregaba tierra virgen para su desmonte y siembra. Otra táctica fue reubicar y recortar las parcelas de los huasipungueros descargando en sus trabajadores parte de la crisis, al estrechar las condiciones de su reproducción, ampliando así la frontera interna de la hacienda.

La creciente cantidad de indios en los predios de hacienda o alrededor de ella, les permitió instrumentar una táctica adicional a los terratenientes: Congelar y disminuir el número de huasipungueros, acrecentando en cambio el número de aparceros, de arrimados y de yanaperos, a los que se les podía imponer relaciones aun más desventajosas que a los huasipungueros. La hacienda no asumía toda la reproducción de esa fuerza de trabajo, ellos más bien se reproducían a partir de su propia unidad doméstica, su relación con huasipungueros o al interior de las comunas, sin que ello le cueste al hacendado que usufructuaba así de una fuerza de trabajo barata para los picos agrícolas. Pero también esta táctica de los hacendados entró en crisis. Los aparceros y arrimados pugnaban por adquirir tierras de la hacienda y convertirse en comuneros; los yanaperos no soportaban las cada vez peores condiciones que pretendía imponerles la hacienda, pugnando también por adquirir tierras, ya no de reversión que se habían agotado, sino de la propia hacienda.

Todas estas tácticas de la hacienda eran tradicionales, en el sentido de buscar soluciones en el marco de los sistemas agrícolas tradicionales y en mantener la renta aumentando los coeficientes de explotación de las relaciones de servidumbre. Sin embargo, la hacienda instrumentó también y al mismo tiempo, salidas modernizadoras basadas en la incorporación de maquinaria, no tanto para aumentar la productividad, sino para ahorrarse fuerza de trabajo; incorporaron fertilización química al mismo tiempo que mantenían la orgánica y comenzaron a experimentar con nuevos cultivos para 
cambiar sus estrategias productivas. Vale decir, no establecieron oposición, como se pensó en el pasado, entre crisis local, conflictividad campesina y búsqueda de modernización pautada y selectiva de las haciendas, en medio de una coyuntura nacional e internacional que dinamizaba esos cambios.

A partir de la década del 60 , los hacendados privilegian la producción lechera, no solo por demandas del mercado, y los estímulos estatales, sino como la mejor opción para arreglar el conflicto con los trabajadores, optando por una estrategia productiva que la habían experimentado largamente.

Agenda de transformación productiva: En la actualidad se cuenta con un proceso de Agenda de Transformación Productiva que ve necesario superar la matriz primaria exportadora extractiva y dirigirnos hacia un mayor desarrollo tecnológico, una diversificación productiva y una diferenciación en términos de productos, mercados y actores, que nos permitan insertarnos en una economía globalizada y aprovechar sus oportunidades.

Busca necesario potenciar las mejores capacidades y recursos: biodiversidad, talentos, capacidades empresariales y activos, a través de estrategias, políticas, programas y proyectos dirigidos a responder los requerimientos y necesidades para aprovechar dichas ventajas. Tiene como misión, fomentar y acompañar un proceso de transformación de la estructura productiva del país, a través de la implementación de políticas y programas que permitan generar competitividad y productividad sistémicas a la vez que de democratizan las oportunidades y el acceso a los factores de la producción, a través de instrumentos específicos.

Considera el rol del Estado en la diversificación productiva, comienza evaluando las diversas fallas del mercado y de gobierno y discute la necesidad de que el Estado supla dichas fallas a través de una intervención que vaya más allá de la dotación de servicios básicos y que implique una participación más proactiva en el ámbito productivo, pero también en el posicionamiento de derechos económicos, sociales y culturales como objeto de las políticas.

La crisis y el nuevo escenario internacional cuestionan los límites de la auto- regulación del mercado y de las políticas selectivas, industriales y sectoriales. La globalización plantea nuevos desafíos para el Estado sobre el fortalecimiento de las políticas integrales, bajo un principio de equidad real o sustantiva, donde el acceso a recursos productivos, el desarrollo de capacidades, el acceso al financiamiento y los mercados y la disponibilidad de infraestructura y tecnología son ejes de la igualdad en esta dimensión.

La agenda tiene como objetivo mejorar la competitividad sistémica de la economía ecuatoriana a través de: La generación de bienes públicos como infraestructura, cambio en matriz energética, talento humano preparado y reduciendo los costos de transacción en la operación de las empresas. 
La estructura productiva del Ecuador, a pesar de haber desarrollado en la década de los setenta y comienzos de los ochentas una importante base industrial, no logró transformar su matriz productiva caracterizada por una fuerte concentración y una alta dependencia del sector petrolero (Tabla 3).

\section{Table 3}

Sectores estratégicos de la agenda de transformación productiva.

Recursos naturales
Alimentos
Cadena Agroforestal
Energías Renovables

Servicios
Turismo
Automotor
Servicios Logísticos
Confección y calzado

\section{Tecnología}

Petroquímica

Metalmecánica

Software

Farmacéutica

Plástico y Caucho

Fuente: MCPEC.

Table 4

Comparativa del Ecuador respecto a otras economías del mundo (exportaciones).

Posición
1
2
3
4
5
70
71
72

País
China
Alemania
Estados Unidos
Japón
Francia
Luxemburgo
Ecuador
Bangladesh

Exportaciones (miles de millones
USD)
1,506
1,337
1,270
765,2
508,7
17,82
17,37
16,24

La situación de la estructura productiva está asociada a las características del empleo, para el caso ecuatoriano cerca del $70 \%$ de la mano de obra empleada se encuentran en actividades basadas en recursos naturales y de bajos niveles tecnológicos [20]. El aumento de la proporción de trabajadores en sectores de baja productividad repercute en los niveles de equidad de la sociedad, ya que tiende a generar una distribución más desigual de los salarios a favor de un grupo más reducido de trabajadores, que tienen más capacidades y están mejor insertos en actividades de mayor productividad. Se han definido 8 pilares de la Agenda de Transformación Productiva:

1. Cambio de Matriz Productiva;

2. Reducción heterogénea estructural;

3. Democratización de los recursos; 
4. Talento humano;

5. Sistema integral para innovación y emprendimiento;

6. Productividad sistémica;

7. Crecimiento verde;

8. Cambio cultural e imagen país.

Table 5

Posición del Ecuador en exportación en Sudamérica (Año 2010).

\begin{tabular}{ll} 
País & Exportaciones (miles de millones USD) \\
\hline Brasil & 200 \\
\hline Argentina & 69 \\
Venezuela & 65 \\
Chile & 64 \\
Colombia & 40 \\
Perú & 36 \\
Ecuador & 17 \\
Paraguay & 8 \\
Bolivia & 7
\end{tabular}

La exportación en el Ecuador: El cambio de matriz productiva a más de un desarrollo endógeno debe responder fuertemente a las necesidades de exportación, pues muchos productores detectan esta opción como elemento estratégico para colocar sus productos. Para ello se presenta una mirada rápida respecto del Ecuador y las exportaciones.

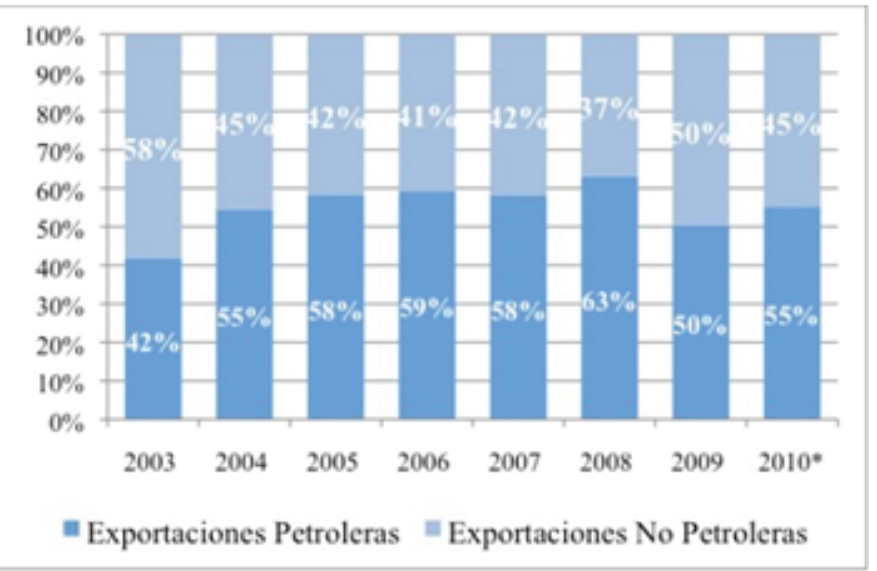

Figure 1

Comparativo de exportaciones petroleras y no petroleras del Ecuador. Fuente: MCPEC. 


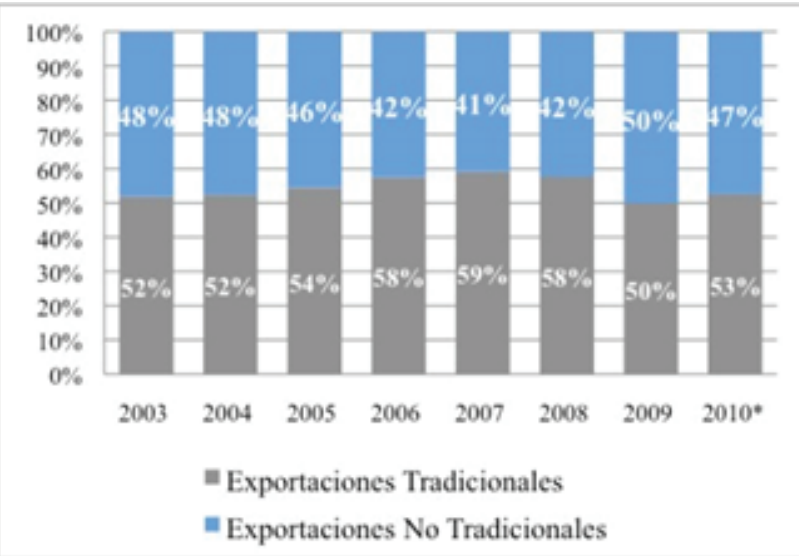

Figure 2

Comparativo de exportaciones tradicionales y no tradicionales del Ecuador. Fuente: MCPEC.

En un primer momento, al revisar las estadísticas en el index mundi la posición del Ecuador entre los 222 países en una variable que proporciona el monto total en dólares americanos de las exportaciones de mercancías sobre una base c.i.f (costo, seguro y flete), se identifica que nuestro país se ubicó en el año 2011 en la posición 71 y sus exportaciones superaron los $\$ 17$ mil millones (Tablas 4 y 5 ).

Las exportaciones alcanzadas por el Ecuador, son totales, es decir las petroleras y las no petroleras, y de aquellas exportaciones no petroleras, hay que diferenciar las tradicionales (banano, café, cacao, etc.) y las no tradicionales (aceites esenciales, frutos tropicales, caña gadua, manufactura de cuero, manufactura de metales, etc.). Obsérvese las Figuras 1 y 2 que denotan un comparativo desde el año 2003 hasta el año 2010 [21].

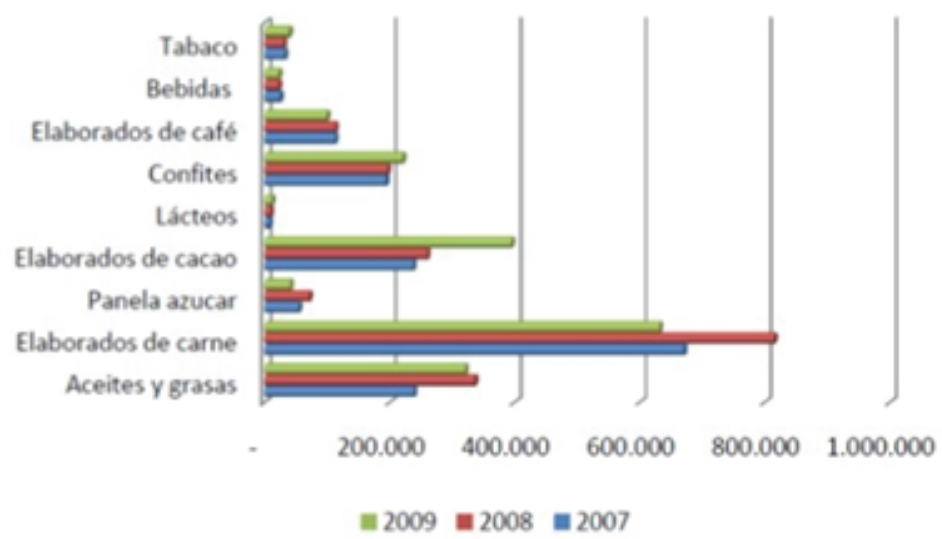

Figure 3

Exportaciones Agroindustriales 2007-2009 (miles de dólares). Fuente: BCE - Bases NANDINA de Comercio Exterior. 


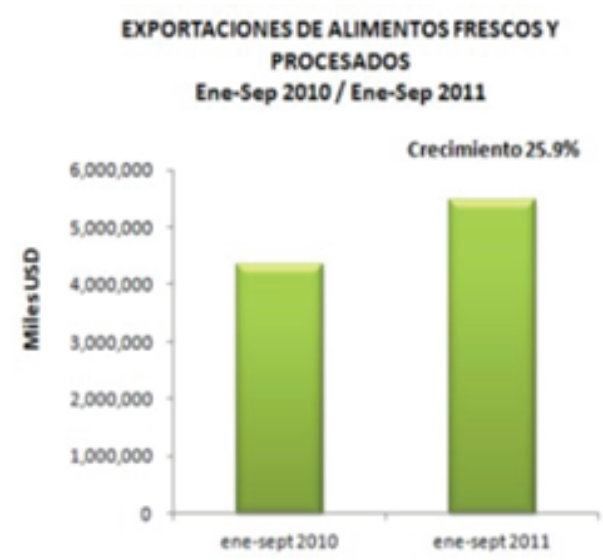

\section{Figure 4}

Crecimiento de exportaciones de alimentos frescos y procesados. Fuente: MCPEC.

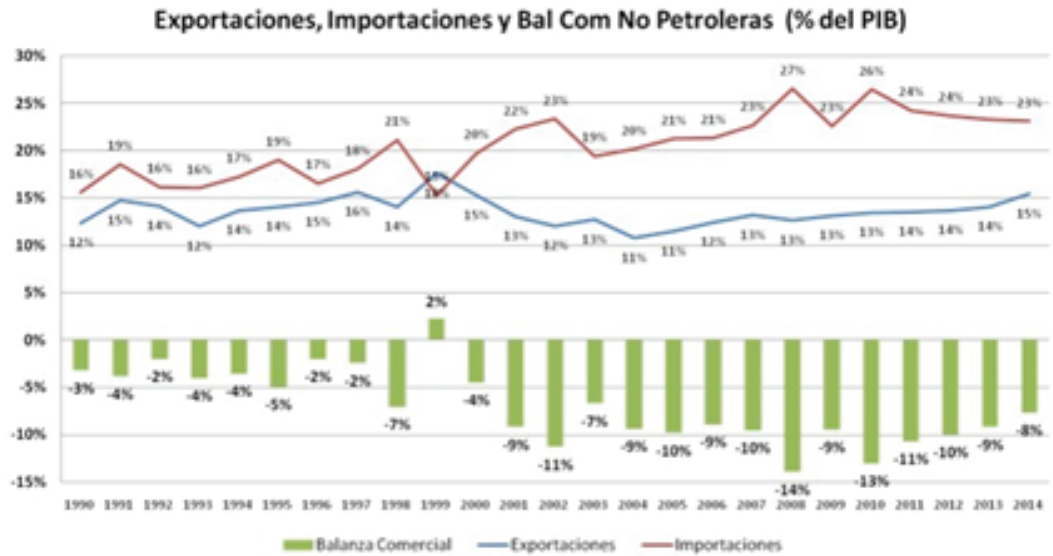

Figure 5

Análisis de la balanza comercial no petrolera al 2014. Fuente: MCPEC.

Según Luis Luna Osorio [22], las exportaciones totales han crecido de 12.728 millones de dólares en el 2006 a 17.500 millones en el 2010, elevándose en más de una tercera parte. El aumento ha sido significativo. Pero, la razón no está en el aumento del volumen y en el mejoramiento de la estructura prevaleciente, sino básicamente en el alza del precio del barril de petróleo exportado, que pasó de USD 50,75 en el primer año a USD 71,93 en el segundo, con un aumento del 42\%; y, en el crecimiento 2006-2010 del valor de las exportaciones de banano (68\%), camarón (44\%), flores (39\%), cacao (144\%), todas exportaciones primarias.

Los rubros de mayor significación en el 2010 fueron los derivados de petróleo (19\%), las manufacturas de metales (vehículos especialmente) (18\%) y los otros elaborados de productos del mar (16\%), que en cada caso superaron los USD 600 mil dólares. 
De las exportaciones agroindustriales reflejamos en la Figura 3 para representar donde se ubica la fuerza de exportación. Entre los productos de exportación se identifica un crecimiento respecto de los alimentos frescos y procesados (Figura 4).

El mercado creciente de oportunidades de exportación requiere de una gestión de comercio con énfasis en la diversificación de mercados de destino y de productos de exportación, donde los estudios de mercado, la información logística, estadísticas y el análisis del comercio internacional permitirán definir las oportunidades y amenazas para el ingreso de productos nacionales en mercados extranjeros (Figura 5).

\section{Conclusiones}

Promover productos innovadores en el marco de la competitividad y de diversificación de la matriz productiva en la provincia de Chimborazo, esto implica un acercamiento a un concepto aún desconocido por muchos actores locales. Es por eso que integrarlo a la cotidianidad de la gestión provincial significa un aprendizaje progresivo y dinámico, donde deben participar los agricultores en el que el diálogo permanente para nutrir metas y aspiraciones de mercado, tanto en el corto, mediano y largo plazo.

Un proceso de acercamiento a mercados es complejo, requiere numerosos condiciones que implica la adopción de prácticas que muchas veces son difíciles de aplicar y que involucran cambios importantes en el proceso de los actores. No obstante, es posible considerar avanzar en esfuerzos de incorporar la innovación en las cadenas de producción de los productos alternativos.

Los procesos de innovar y manejar productos alternativos implica familiarizarse primeramente con un nuevo lenguaje, un proceso de cambio en la mentalidad, nuevas forma de producir y de establecer relaciones con públicos de interés.

No cabe duda que el aprovechamiento de nuevos productos reportará ventajas competitivas a la provincia, y numerosos beneficios a los productores. Siempre y cuando se maneje una productividad e innovación que brinde impactos económicos, sociales y ambientales positivos.

La innovación es el eje central para poder establecer un nivel de productos alternativos lo suficientemente estratégicos para el mercado de consumidores de nivel competitivo.

Las señales u orientación al mercado generadas en el presente estudio son de naturaleza de comportamiento, más no representanta aún la generación de inteligencia de mercado que permita determinar la fiabilidad y validez de cada producto.

El mercado tiene sus dinámicas y exigencias, por lo tanto el primer paso es la adaptación a sistemas de innovación institucional, productiva y tecnológica que permita 
desarrollar los sentidos para conectarse con los mercados, especialmente los justos e inclusivos.

\section{Agradecimientos}

Los autores agradecen al GAD provincial de Chimborazo por el financiamiento para esta investigación, a la Facultad de Recursos Naturales de la ESPOCH por brindar las facilidades para el levantamiento de la información, a quienes revisaron este trabajo nuestro agradecimiento por sus valiosos comentarios.

\section{Conflicto de Intereses}

Los autores resaltan que no existen conflictos de intereses particulares con las entidades tanto científica como financiadora que pudiesen afectar directa o indirectamente a los resultados obtenidos.

\section{References}

[1] Aguirre C. Resultados de investigaciones silviculturales en el ecuador, Proyecto INERNITTO PD 13891 (F). Quito: Ecuador; 1993.

[2] Cañadas L. El mapa bioclimático y ecológico del ecuador, MAG-PRONAREG. Quito; 1983.

[3] Maldonado AM, Méndez C, Santillán F et al., Experiencias en gestión y desarrollo local. Centro de Investigaciones CIUDAD; 2005.

[4] SENPLADES. Plan Nacional para el Buen Vivir. Quito; 2013.

[5] Código Orgánico de la Producción. Comercio e Inversiones, Registro Oficial Nro. 452. Quito; 2011.

[6] Tapia C, Zambrano E, Monteros A. Informe nacional sobre el estado de los recursos filogenéticos para la agricultura y alimentación ecuador. instituto nacional autónomo de investigaciones agropecuarias INIAP; 2008.

[7] Baquerizo MIS. El Rol del Comercio Internacional en el Crecimiento Económico del Ecuador: Antecedentes y Perspectivas. 2003.

[8] Kotler P, Armstrong G, Cámara D, Cruz I. Marketing. Prentice Hall; 2004.

[9] Andrade F. Comercio justo o economía solidaria, AFECE. 2008;49:103-108.

[10] Andrade MR, Gutiérrez IS. Productos y servicios sostenibles una opción de exportación para el ecuador, ecuador comercio exterior. Universidad del Azuay; 2010 
[11] Pelayo CM. La Competitividad. Monografias 2011. Disponible en: https://www. monografias.com/trabajos/competitividad/competitividad.shtml

[12] Agenda de Matriz Productiva. Quito: Ministerio de Coordinación de la Producción Empleo y Competitividad; 2013.

[13] Chiriboga M. Agricultura y globalización: Impacto sobre los Pequeños Productores, los Trabajadores Rurales y los Consumidores. Quito: RIMISP; 2007.

[14] Berdegué JA, Escobar G. Innovación Agrícola y Reducción de la Pobreza. RIMISP; 2001.

[15] Ministerio de Coordinación de la producción, empleo y competitividad. Programa INNOVAECUADOR. 2012. Disponible en: www.innovaecuador.gob.ec

[16] Mytelka. Local systems of innovation in a globalized world economy. Industry and Innovation. 2000;7(1):15-32.

[17] Larrea C. El banano en el Ecuador. Quito: CEN. II; 1987.

[18] Abad G. Modernizador de la via. Farmer del desarrollo Agropecuario. 1970.

[19] Larrea C. El banano en el Ecuador. Quito: CEN. III; 1987.

[20] Organización de las Naciones Unidas para el Desarrollo Industrial. Informe Anual 2007. 2007

[21] Brito D. Agro-exportación de productos no tradicionales. 2002. p. 1-20

[22] Osorio LL. Solo "C" que nada "C". Las Exportaciones no Tradicionales del Ecuador y el Desarrollo Nacional. 2011. Disponible en: http://solocquenadacbyllunao.blogspot. com/2011/08/las-exportaciones-no-tradicionales-del.html 